\title{
Confine o frontiera? \\ La Liguria di Francesco Biamonti
}

\section{Giorgio Bertone}

Università di Genova

\begin{abstract}
Di Francesco Biamonti (San Biagio della Cima, Imperia, 1928-2001) recentemente scomparso restano quattro romanzi omogenei quanto a temi e stile, asciutto e scarno, e ben radicati nella geografia dell'entroterra dell'estremo Ponente ligure. Uomo e scrittore di confine o di frontièra? Definendo, sia pure in via provvisoria, con "confine» una situazione geoculturale ben delimitata da una linea che marca più o meno nettamente la divisione tra «dentro» e "fuori», e con "frontiera» una fascia mobile che pulsa e si apre di continuo a nuovi apporti, Giorgio Bertone propende per l'arruolamento di Biamonti nella prima categoria, nonostante le evidenti proiezioni dello scrittore ligure verso la cultura, il paesaggio, la lingua francese. In aquesta dialettica tra "confine» e "frontiera», scandita anche su altri exempla illustri, si scava e definisce meglio, al di là della piccola mitologia sorta intorno al personaggio Biamonti, il senso della sua arte e del suo inconfondibile stile.
\end{abstract}

Parole chiave: Francesco Biamonti, frontiera, Liguria, paesaggio e letteratura.

\section{Abstract}

On his recent death, Francisco Biamonti (Saint Biagio della Cima, Imperia, 1928-2001) left four novels, homogenous in terms of theme and style and the dry, inhospitale landscape profoundly located in the far western hinterland of Liguria. Man and border of the frontier writer? Provisionally defining, border in terms of a limited geo-cultural situation, a line that more or less clearly marks the division between «inside» and «outside», and "frontier» in terms of a mobile construct or fa ade that pulsates and is continually open to new contributions, George Bertone inclines toward placing Biamonti in the first category, in spite of the Liguria writer's evident connections with French culture, landscape, and language. He pursues the dialectic between «border» and «frontier», also demostrated with other notable examples, refines the definitions of the terms and also examines the small body of mythology that has arisen around the personality of Biamonti, his art and unmistakable style.

Key words: Francesco Biamonti, frontier, Luguria, landscape and literature. 
La recente scomparsa di Francesco Biamonti, il 17 ottobre 2001, chiude con un taglio netto e doloroso, insieme con la parabola esistenziale, l'esercizio di alta scrittura, che resta, nell'ora più ferma, ben confinata in quattro romanzi (tutto il rimanente, salvo sorprese, andrà rubricato nella categoria solo indicativa e provvisoria del «minore», se non proprio nelle curiosità); ${ }^{1}$ quattro tempi di un'unica ricerca stilistica, retorica e figurativa, scanditi in stagione tarda (classe 1928, il nostro Biamonti) ${ }^{2}$ come il frutto di un lento accumulo, dell'abbandono di lontani tentativi, e di una accurata maturazione esente da troppo pressanti ansie editoriali (1983: L'Angelo di Avrigue; 1991: Vento largo; 1994: Attesa sul mare; 1998: Le parole la notte). ${ }^{3}$

Fortemente radicato da una fedeltà di sguardi e di passi nella sua terra, il tetrastico di romanzi può vantare una sua immediata, certificabile denominazione d'origine geoletteraria: al confine tra Italia e Francia, al confine tra terra e mare, al confine tra monti aspri (prealpi e vere montagne alpine, il ripido e lo scosceso orografico come segno primario dei luoghi) e la piana mediterranea, al confine tra mondo contadino e cultura cittadina (turistica, urbanistico-architettonica e, in tutti i sensi, di traffici), al confine tra vita antica, atavica e moderne correnti cosmopolite, plurietniche. È persino un luogo deputato, il confine: nel secondo romanzo, Vento largo, in particolare, l'invisibile ma concreta barriera doganale tra Francia e Italia attraverso cui i passeurs conducono gli emigranti clandestini di tutte le razze, segue una linea che separa la «sua» Liguria

1. Il che non significa che non esistano interventi o scritti d'occasione che illuminino più vivamente i versanti delle opzioni essenziali; o che costituiscano una sorta di importante corollario dei testi maggiori.

2. Francesco Biamonti è nato a San Biagio della Cima, nell'entroterra di Vallecrosia, il 3 marzo del 1928. I suoi amori furono sostanzialmente le piante, i libri e la pittura; la bellezza femminile e l'erotismo rimangono in una zona umbratile, eppure di evidente forte pressione, una zona di frontiera intima, in parte imperscrutabile, per dirla con uno dei paradigmi che ci siamo scelti. Leggenda editoriale è la sua attività di coltivatore di mimose (di cui non amava il colore: «Il loro giallo è fatuo, ignaro delle tenebre del mistero, la cifra dei fiori europei»), mentre andrà detto che dopo aver vagabondato in Spagna e soprattutto in Francia, fu bibliotecario all' Aprosiana di Ventimiglia (1956-1964, all'incirca). Non amò la biografia e l'autobiografia: «[domanda] Se io domandassi di raccontarmi qualcosa della sua vita, lei cosa risponderebbe? [risposta] Mi piace non dire niente. Io sono da cancellare. La mia vita non conta nulla; i miei natali non hanno importanza; il mio paese è insignificante. Si fa della letteratura perché non si è contenti della propria vita. Scriva che non si sa nulla. Che sono stato abbandonato da degli zingari di passaggio. Che sono un autodidatta. Che ho fatto le scuole dei preti di Don Bosco, che almeno insegnavano bene il latino [si diplomò in ragioneria]" («Intervista», in Paola MALLONE, "Il paesaggio è una compensazione». Itinerario a Biamonti con una appendice di scritti dispersi, Genova: De Ferrari, 2001, p. 50; la frase in titolo, «il paesaggio è una compensazione», è l'estrapolazione da una serie di dichiarazioni e riflessioni dell'autore stesso ben più complesse; isolata, tradisce l'alta funzione che il paesaggio assume nella sua opera). Che poi lo si debba proprio prendere alla lettera in questa sua richiesta, ben mimata, di annullamento biografico, non è detto, anche se ragioni affettive e consonanza di giudizi sulle «vite» porterebbero lì per lì ad assecondarlo fino in fondo. Indirettamente vuole suggerirci che i suoi romanzi sono già per conto loro fortemente autobiografici.

3. Tutti editi da Einaudi. 
ponentina dalla «sua» Provenza, ovvero i suoi territori non solo culturali. Una sorta di osmosi dunque? Al di là di una demarcazione netta ovvero un limes, una frontiera che pulsa nel cuore bivalve di un uomo che la vive come un doppio nutrimento di sangue e un unico battito vitale?

Ogni scrittore, soprattutto se vive intensamente vicino a una dogana, assume il confine come un dato imprescindibile e lo sconvolge creando una nuova frontiera. Si tratta di verificare con mano quale delle due configurazioni infine prevalga. ${ }^{4}$

\section{1.}

Francesco Biamonti è stato pure un affascinate parlatore e atteso conferenziere. Partiva in sordina, con fare volutamente impacciato, e come nell'atto di una continua scusa, quasi a confermare quel mito del contadino o del «coltivatore di mimose» che Calvino e l'Einaudi gli avevano appiccicato addosso. Poi sciorinava, quasi senza farsene accorgere e con un tono di voce appena più sostenuto, una serie di citazioni, italiane e francesi. Ben dosate, calibrate, offerte dalla voce roca anche se avvolte da un tono di excusatio finto-vernacolare anche nel timbro e nella cadenza; offerte come si offre il frutto di un proprio orto o giardino privato (orto e giardino, si badi, coincidono nella poca terra del contadino ligure). Erano i suoi autori: i poeti Paul Valery, René Char, e Baudelaire ( Ho trovato su una bancarella, quand'ero adolescente, Les fleurs du mal. Ho cominciato con Baudelaire - avevo sedici anni - con il simbolismo francese» $)^{5}$, gli scrittori Camus, Sartre, gli esistenzialisti. S'aggiungano i filosofi puri alla Merleau-Ponty (con, dietro, Husserl). ${ }^{6}$ Insomma, le letture della lunga carriera di autodidatta di chi fin da giovane andava «quotidianamente a Mentone» a spulciare e far bottino di libri. E di cultura francese e provenzale il testo è impregnato. Impregnato, eccome, ma non allineato su un filone preciso o su un'idea o ideologia predominante. Nell' Angelo c'è persino un pastore che parla provenzale (" - Pregatz per nos, — ritmò, — que Deu nos faga bons crestians e que non aduga, nos, pastre, nauchié e gent de mas, a bona fin» ${ }^{7}$, con una nobile inflessione che richiama, alla lontana, il Dante del XXVI del Purgatorio).

4. Per un esame di un'altra frontiera, quella tra Italia e Svizzera, e alcuni spunti di indagine anche teorici (ma il termine poi assume i più diversi significati fino a diventare metaforico e onnicomprensivo) si veda: Jean-Jacques MARCHAND (a cura di), Varcar frontiere. La frontiera da realtà a metafora nella poesia di area lombarda del secondo Novecento, Roma: Carocci, 2001. E ancora, sul versante filosofico Achille VArZI e Luca Morena (a cura di), Oggetti fiat, numero monografico della Rivista di estetica, 20, XLII, 2002.

5. "Io andavo quotidianamente a Mentone, dove c'era una libraia, appassionata di letteratura, che teneva tutto. Leggevo "Combat", che era un giornale al quale collaborava Camus e Camus era un grande ammiratore di Char [...]. Char per me è stato fondamentale.» ("Intervista», in "Il paesaggio è una compensazione» [...], cit., p. 53).

6. Me ne parlò personalmente.

7. Francesco Biamonti, L'Angelo di Avrigue, cit., p. 116. 
Basta però questo perché si possa parlare in re, nel corpo dei libri realizzati, di un'idea di frontiera come luogo suscettibile di espansioni e contrazioni, in movimento, dove le culture si rincorrono e contrastano, si sfidano in vista — eventualmente - di un traguardo, della realizzazione avventurosa di un qualche ideale o terra promessa del futuro o del passato? $\mathrm{O}$, invece, sarà da dire che i suoi francesi come i suoi italiani (Pavese, Tozzi, Pea, Montale, Sbarbaro; ma anche Boine, per dirne uno viciniore) ${ }^{8}$ fanno parte più o meno allo stesso titolo di un bagaglio a lungo accumulato, un nutrimento a lungo ruminato, da cui infine sono scaturiti personaggi, trame, paesaggi, occasioni che $s$ 'iscrivono in una mappa affatto personale, topograficamente - cioè psicologicamente e culturalmente - ben stabilizzata a delimitare uno spazio in cui l'io sotteso ha la postura statica, antica, della figura assisa che pensa?

2.

Peculiare, - come hanno compreso fin da subito i suoi lettori non solo liguri -, e tutta personale è la cartografia di Biamonti. ${ }^{9}$ Si direbbe che il paradigma geografico non possa escludersi dalla visitazione critica, a qualsiasi livello, del suo universo territoriale-mentale (e della sua scrittura, s'intende). Già il titolo del primo libro offre una spia di un programma preciso in quel toponimo, Avrigue. ${ }^{10}$ In breve e con un certo tasso di approssimazione diremo così: sulla linea di confine politico reale che sale più o meno dai Balzi Rossi (sul mare, un poco a Ovest di Ventimiglia) verso Nord-Nordovest, s'innesta un

8. «[Quella francese e quella italiana] sono due letterature complementari. La Francia ha più rigore di scuola; l'Italia più individualità. Dell'Italia mi piaceva molto il primitivismo toscano, Tozzi, Pea e della Francia mi piaceva la poesia simbolista, poi Camus, Sartre, l'impegno umano e politico, il lirismo "assurdo" di Camus. Sono due scuole di cui mi sono servito. Insomma da qualche parte bisogna sempre partire. Poi anche la poesia di Montale, Sbarbaro, come anche la poesia di Valery hanno influenzato la mia formazione.» ("Intervista», in "Il paesaggio è una compensazione» [...], cit., p. 52). Passi di evidente influenza boiniana sono nel debutto dell' Angelo.

9. Rinvio al mio studio su Biamonti in Paesaggio e letteratura. Liguri e no. Montale, Caproni, Calvino, Ortese, Biamonti, Primo levi, Yehoshua, Lecce: Manni editore, 2001, anche perché mi è servito, per alcuni aspetti, da base per il presente saggio.

10. "Il paese di Avrigue è con ogni evidenza lo stesso che Apricale, l'antico paese della Val Nervia, dopo Dolceacqua e dietro il colle di Perinaldo, che nel dialetto del luogo suona Avrigà. Il latino apricum, "esposto al sole", dà infatti avrigulabrigu (c'è anche una Costa Abrigo sopra Seborga), così come opacum dà $u b a g u$, donde il toponimo diffuso in quella regione: Ubago, appena sotto Pigna, e il Bosco dell'Ubago, la Fascia d'Ubago, Rio Ubaghi, ecc. mentre "gli ubaghi" per i luoghi in ombra fanno parte a loro volta del lessico di Biamonti (per la dialettica tra l'ubagu e l'abrigu si ricordino le pagine di Calvino, in Adelphiana 1971, e quelle di Giorgio Bertone, in apertura e chiusura del suo saggio, nel volume dedicato alla Liguria, nella Storia d'Italia Einaudi). La forma Avrigue la si direbbe tuttavia forma francesizzata di Abrigà: il che ricorda quel tentativo di rattachement alla Francia delle valli di confine, nel '45, al quale si accenna in $P N$ [Le parole la notte, p. ] 85 e 116 [...]. Non dunque Apricale, ma una forma francese, Avrigue, che s'innesta su quella dialettale, Avrigà.» (Enrico FENZI, «Toponomastica e antroponomastica in Biamonti», il Nome del testo, II-III, 2000-2001, p. 61). 
paesaggio geoculturale tutt'affatto personale che dovrebbe incentrarsi su San Biagio della Cima, il paese di fondovalle di Biamonti, ${ }^{11}$ il qual paese sta però due valli più a Est, — nella Val Nervia, dopo la Val Roja — , e invece a forza di traslazioni di luoghi, toponimi e vedute si innalza di quota e da lassù si schiude in un ideale ventaglio aperto a Ovest e Nord-Ovest, che include i monti, italiani e francesi, esclude le città della costa italiana a Sud, contempla, nominandole più volentieri, quelle della costa francese fino a Tolone e Marsiglia. E oltre: fino alla Catalogna e Barcellona, se possibile. Del resto non siamo abituati a considerare che lo sguardo della mente dell'intellettuale desiderante possa agevolmente superare l'ostacolo della curvatura terrestre fino dai tempi in cui Petrarca su un monte provenzalissimo come il Ventoux «vedeva» i Pirenei? ${ }^{12}$

Con tutta evidenza Biamonti inclina e flette le sue coordinate verso Nizza (la Baie des Anges o la Baia degli Angeli, indifferentemente), verso Cannes e le due isole, Saint-Honorat e Sainte-Margherite; e verso i monti dell'una e dell'altra parte (Cima Marta). Cancella il litorale italiano come luogo della «speculazione edilizia» (ben sapendo che già l'aveva cancellato il «suo» Calvino: «Io ero della Riviera di Ponente; dal paesaggio della mia città - San Remo - cancellavo polemicamente tutto il litorale turistico - lungomare con palmizi, casinò, alberghi, ville - quasi vergognandomene», ${ }^{13}$ con quel che segue di descrizione della risalita verso i monti all' inseguimento del suo paesaggio), del traffico maleodorante di auto, e dei traffici malavitosi; anche se, quanto a macrocriminalità (investimenti delle varie mafie non solo italiane) e microcriminalità nelle Passeggiate, sa bene che la Côte d'Azur non ha niente da invidiare alla Riviera. Làtitano dunque, censurati, i toponimi di quest'ultima, che saranno Bordighera o Ventimiglia. Se la più terribile, Sanremo, è nominata una tantum, sarà per bollarla per sempre:

Cose di cui era meglio non parlare, cose che aveva stentato a credere: ebrei in fuga, derubati e buttati in mare da un barcaiolo nel '38 o nel '39, pastori sgozzati nei casolari da gente che transitava. - Sarebbe meglio non stare sui confini, — si limitò a dire. — O forse tutto il mondo è uguale.

11. «San Biagio della Cima m. 1OO, ab. 840, cosiddetto perché sovrastato dalla precipite parete di arenaria del monte $S$.Croce, sulla cui sommità si trova una cappella in rovina. Il borgo è disposto lungo il crinale che dalla dorsale della Val Nervia scende verso levante, e si accentra attorno alla parrocchiale dei Ss. Sebastiano e Fabiano, chiesa barocca del 1777 [...]. La strada, sempre seguendo il corso del torrente, che assume qui il nome di Verbone (le coltivazioni floricole cedono il posto a quelle viticole per la produzione di vino Rossese), raggiunge, km 8.5, Soldano» (AA.VV., Liguria, Milano: Touring Club Italiano, 1982 (6a ediz.), p. 547).

12. Per un'analisi paesaggistica e culturale dell'episodio petrarchiano e per una discussione generale sul concetto di paesaggio in letteratura rimando al mio Lo sguardo escluso. L'idea di paesaggio nella letteratura occidentale, Novara: Interlinea, 1999, p. 126-132.

13. Italo Calvino, Prefazione 1964 [al Sentiero dei nidi di ragno], in ID., Romanzi e racconti, edizione diretta da Claudio MiLANINI, a cura di Mario BARENGHI e Bruno FALCETTO, vol. I, Milano: Mondadori, 1991, p. 1188. 
— Io sto meglio qui che a via Martiri a Sanremo, _ disse Astra.

— Non se ne parla nemmeno. Quella è una galera. ${ }^{14}$

Lo sguardo del desiderio e della ricerca del discrimine deforma persino le linee orografico-meteorologiche: in Attesa sul mare pur di legare Saint-Malo e il Golfo di Biscaglia, attraversato dalla rotta dell' Hondurian Star, la nave carica di armi clandestine, alla sua terra, fa nascere lassù l'adorato vento provenzale del Mistral:

Punto stimato: al largo del golfo di Biscaglia. S'incrociavano nel cielo delle lame: dorate e argentee. Si formava il Mistral: sarebbe arrivato a Tolone prima della nave. ${ }^{15}$

Un Mistral da legenda personale che nasce e muore dove vuole l'autore, unifica Provenza, Francia, il loro suolo, ai crinali dell'entroterra ligure e al Mediterraneo sui cui s'affacciano.

3.

Gli spostamenti e le torsioni imposte alla carta geografica svolgono un precisa intenzione di apertura a una frontiera ideale che potrebbe persino innestarsi su un episodio storico, il rattachement del ' $45 .{ }^{16}$ Dell'occupazione da parte delle truppe francesi dei territori di oltreconfine italiani si parla in Le parole la notte ${ }^{17}$ attraverso la figura (inventata) di un ufficale che partecipò a quell'episodio, Albert Corbières:

- Sono contento di conoscerla. Vorrei notizie del suo paese. Potrei dirle che l'ho amato e che lo ricordo ancora pieno di rose.

— Quando c'è stato?

- Nel ' 45.

- Nel dopoguerra?

— Possiamo anche dire così. Sono venuto a conquistarlo, o a liberarlo, se preferisce.

14. Le parole la notte, cit., p. 74-75.

15. Il Mistral si forma tra il Massiccio centrale e le Alpi, nella valle del Rodano, da lì, dal centro giunge alla foce a Marsiglia e si apre a ventaglio sul Mediterraneo. Tre righe più indietro, durante il dialogo fra naviganti, si legge: «- Stanotte, poco prima dell'alba, c'era pieno di battelli-fanale a cinquanta gradi ovest. / - I soliti pescatori portoghesi.», dove la frase «a cinquanta gradi ovest» suona di primo acchito molto marinaresca, invece è nauticamente priva di senso, perché non può voler dire "cinquanta gradi a ovest" che è una distanza enorme $(50 \times 60$ miglia). Si direbbe che a bella posta Biamonti abbia disseminato il testo di espressioni nautiche altamente improbabili e inverosimili, incurante degli eventuali consigli a portata di mano (il fratello "marittimo») per rimarcare, lui così preciso nella terminologia botanica e terrestre, la sua indifferenza o estraneità al mondo nautico, e dunque al viaggio.

16. Sul rattachement insiste Enrico FENZI, «Toponomastica [...]», cit., p. 73-74; il quale propone un'interpretazione dell'opera di Biamonti in chiave «etica e politica» (Ibid., p. 71).

17. Alle p. 16, 85, 115-116, 158-159, 181. 
- Credo che non sia più come lo ricorda.

- Certamente no. Nulla in Europa è più come allora. Era un'Europa carica di rovine. Ero sottotenente e al suo paese mi sono trovato bene. Argela. Noi l'avevamo chiamato Argèle-Les-Rosiers.

- Grazie per quel bel nome. Chi l'ha trovato?

- L'ho suggerito io. Dovevamo trovare i nomi per il rattachement.

- Annessione a che?

- Alla Provenza, alla Francia. Lei non era d'accordo?

- Ero ragazzo. Mi ricordo come in un sogno. Un mio zio era francofilo fervente. Quando era caduta Parigi aveva pianto.

- Dunque non eravate contro di noi.

- Jamais de la vie! Ma potevate cambiare tutti i nomi che volevate, sarebbero sempre rimasti paesi della fame: quattro rocce e nulla più. ${ }^{18}$

Storicamente non è più che un breve episodio, mal condotto, caratterizzato dalla strana e ambigua figura dell'agente francese, falso prete, l' «abbé Pontremoli». ${ }^{19} \mathrm{Nel}$ romanzo i ricordi dell'ufficiale sono legati al sentimento di tutta una vita trascorsa a inseguire sogni di patrie («aveva creduto in troppe cose»), in ultimo quella sionista; e soprattutto alla ricerca di un ubi consistam definitivo ("un posto dove morire»). Che però non troverà in Italia, ma - scelto il suicidio per evitare il calvario della malattia - nella sua Francia («versate le mie ceneri, vi prego, a Bargème sotto la montagna di Brouis»). Insomma, ritorno definitivo alla sua terra. E ritorno cui risponde simmetricamente Leonardo con la sua mente volta meno a coltivare una vena di nostalgia per un altrove, che a ruminare la gnomica della omologazione generale: in fin dei conti dappertutto è uguale, ormai. Come avevamo già letto: «Sarebbe meglio non stare sui confini, — si limitò a dire - $\mathrm{O}$ forse tutto il mondo è uguale». Che è un po' il Leitmotiv: «Qui o altrove non cambia nulla. È tutto un mondo edificato sulle rovine e sui delitti.» ${ }^{20} \mathrm{E}$ ancora, a proposito del personaggio francese: «Leonardo pensava che Corbières era venuto a cercare un posto dove morire e s'era trovato davanti un mucchio di problemi: Alain sconfitto, una costa assassinata, un paese quasi distrutto». ${ }^{21}$

Ecco uno stralcio di dialogo tra l'(idealmente) autobiografico Leonardo e Veronique:

- Com’è Marsiglia? — chiese Leonardo.

- Marsiglia è sempre Marsiglia, il mare entra.

- Comè la città, sta cambiando?

- Si sta meglio qui, su quest'altura.

18. Ibid., p. 85.

19. «- Mi pare strano che fosse un prete. Girava con una bella donna e fumava come un turco. Malgrado il sottanone, camminava con un'agilità che era difficile stargli dietro. / Leonardo ascoltava. "Mi dite cose -pensava- che ormai non servono più e che mi mettono fuori strada."” (Ibid., p. 116).

20. Ibid., p. 74.

21. Ibid., p. 181-182. 
Veronique si alzò e fece segno a Leonardo di seguirla.

[...]

— Vede, — disse Corbières, — Marsiglia decade. Non è più la capitale d'oltremare. Potremmo brindare al suo passato, alla fine di un mondo. Ormai è solo un porto di petroliere. ${ }^{22}$

Quella è la sigla. La sigla, se non proprio della rassegnazione, dell' «ormai». E dove regna l'«ormai» non c'è «frontiera».

4.

Certo, l'insediamento e l'autoinvestitura dell'uomo che ha deciso di abitare il confine ("Vi sono due Ligurie, — pensava, — una costiera, con traffici di droga, invasa e massacrata dalle costruzioni, e una di montagna, una sorta di Castiglia ancora austera; io sto sul confine», ${ }^{23}$ è sempre Leonardo che parla fra sé) si alimenta di una frontiera vagheggiata, virtuale, e impossibile. Ma infine l'accertamento della parificazione e omogeneizzazione del mondo moderno — in una sorta di piccola teodicea gnomico aforistica, ma fedelmente laica, ch'è lo stilema mentale di Biamonti — , impedisce alla "frontiera» di accedere alla forza riscattante di un mito che la renda feconda e la proietti su una qualche méta, fosse pure un miraggio. Corbières viene sepolto nella sua terra. Con lui si seppelliscono sogni e avventure dello spirito. Non c'è futuro neppure come iterazione formale di un passato che poteva includere speranze. Ognuno torna al suo confine. Al suo confino esistenziale. L'ultimo capitolo chiude con il furtivo funerale al cimitero e la sepoltura delle ceneri dell'ex-ufficiale.

«Frontiera» e "confine» non smettono tuttavia di intersecare le loro linee, dinamiche e virtuali le une, statiche, consolatorie e radicate nel suolo, le altre. Almeno questo si potrà dire: il tramonto di un possibile mito di una patria «altra» (Biamonti oltretutto, non si dimentichi, scrive il primo libro a 55 e l'ul-

22. Francesco Biamonti, Le parole la notte, cit., p. 109. Il dialogo continua coinvolgendo un passato in cui, per via di trasmigrazioni e di lavoro la comunicazione con la terra di Francia poteva essere intensa e preannunciare qualcosa di nuovo: «- Brindiamo [dice Leonardo sempre a proposito di Marsiglia] ai suoi scogli, alle sue rocce bianche, ai suoi castelli sulle onde. / - Allora lei la conosce. / - Gli argelesi ci sono sempre andati a lavorare. / - Quando? / - Da sempre, come le dicevo. Gli ulivi caricano ogni due anni. E quando non c'erano frutti gli argelesi partivano. A Marsiglia aveva la sua bottega un calzolaio di Argela che sapeva il francese; lo sapeva anche scrivere e faceva le domande per entrare a lavorare sul porto. Ad Argela, nelle cantine, se ne parlava come di una specie di console. Non ricordo il suo nome, un tempo lo sapevo.» Ma il mito della Francia è morto già in Vento largo, nobilmente morto perché nobile e rispettoso ne è il ricordo: "Portare gente in Francia [dice il passeur Varì] gli sembrava un compito nobile. Poi s'era accorto che la Francia che amava era morta da molti anni. Ma questo non lo disse. Mai parlar male della Francia: era uno dei suoi principi. Intere generazioni di Luvaira e di Aùrno erano andate a togliersi la fame, fame e tante altre cose, sul porto di Marsiglia. Scaricatori di bastimenti, camallavano nel mistral» (p. 88-89; già ce n'era un anticipo a p. 22).

23. Ibid., p. 90. 
timo a 70 anni) rende più laceranti i percorsi, gli andirivieni, gli sguardi sulle linee precise e decise una volta per tutte. Se rende più tagliente il Border che sorge ovunque, ovunque ci sia un pretesto figurativo, un contorno, un crinale - , offre pure, in una sorta di risarcimento, la possibilità di installare saldamente la Musa dentro al Genius loci, il luogo circoscritto dal limes e dalle pietre mercuriali. Nessuno confonderà quello di Biamonti con un semplice buen retiro, assimilabile a quello, italiano o straniero, di alcuni suoi personaggi che restaurano il rustico per girare le spalle al mondo (e in cui si sono identificati molti lettori, equivocando).

\section{5.}

La più tipica, la più importante frontiera d'Europa - la cui portata storica è immane, insuperata — è il Mediterraneo. Biamonti non solo l'osserva e lo descrive, lo naviga, almeno virtualmente. Il protagonista dell' Angelo è un marinaio in attesa di un nuovo imbarco, nel finale del libro il Mistral spazza Marsiglia. In Attesa sul mare il marinaio Edoardo lascia i suoi ulivi malati e s'imbarca, come s'è accennato, su una vecchia nave per le coste della ex-Jugoslavia con una partita di armi clandestina nella stiva, suo compagno di traversata è uno spagnolo, gli ordini arrivano - e poi si taceranno di colpo da una misteriosa agenzia di Tolone via radio. Ma la navigazione è poco più che un pretesto, l'eventuale marinaio che è dentro l'autore, sempre visibile dietro lo schermo dell'attore principale, soffre sempre il «male del ferro», il dolore e l'angoscia rilasciati, come la ruggine, dalle lamiere dello scafo. Il tema della fuga porta sempre al medesimo punto di partenza, alla medesima terra: basta un ulivo per gemellarle tutte. Ulivi, piante e pietre sono i medesimi anche dall'altra parte del Mediterraneo: «Passarono un'erta di rosmarini agitati dal vento. Gli vennero in mente rosmarini e api di Pietrabruna, i cespi di lavanda che il cielo rendeva armoniosi ed infuocava». ${ }^{24} \mathrm{La}$ si potrebbe chiamare la sindrome dell'approdo.

6.

Chiamato a rispondere proprio sul tema del Mediterraneo, ${ }^{25}$ in uno dei suoi ultimi scritti d'occasione - non narrativo: ma riflessione e narrazione in lui si intrecciano, epperò si limitano a vicenda - Biamonti assolve così al suo compito:

A guardarlo dalle nostre colline, della Liguria occidentale, sale all'orizzonte come un immenso edificio di luce. Fa sognare partenze, voli supremi. A volte

24. Francesco Biamonti, Attesa sul mare, cit., p. 102.

25. "Mare di luce e di sangue», in Sergio BuOnadonna (a cura di), Finestra sul Mediterraneo. A window over the Mediterranean Sea, Genova: il Melangolo, 2001, p. 67-68. Il testo appare deformato da refusi e, sospettabilmente, da altro (salti di riga?). Correggo ciò che posso, provvisoriamente, ope ingenii. 
è bianco e fa l'effetto di una nuvola; più spesso è di un azzurro che sconfina; se il vento lo ghermisce, appare solcato di cammini, specie di sera. Ma in fondo che mare è? A un'apertura, a una libertà metafisica non corrisponde una realtà geografica: è quasi un lago e le sue rive sono state spesso insanguinate e lo sono anche adesso. Su coste di sabbia o di roccia si svolgono faide politiche e religiose, lotte d'intolleranza monoteista. Possibile che come dice Freud non si possa vivere senza un dio a contatto del deserto? Dio personificazione dell'eterno e del padre primordiale. Viene da dire con Camus: beati gli orfani. Aver perduto gli dei greci e il dio cristiano è un privilegio che rende liberi e soli con la propria coscienza. Rende beninteso anche tristi e responsabili. È un mare che il più delle volte risplende e il suo bordo lontano sembra versarsi altrove per rifrazione di orizzonte. Montale lo ha chiamato «antico» [...] e avrebbe voluto carpirne la voce e tradurla in "balbo parlare». [...] Camus ha sentito l'orgoglio, l'intima felicità di averlo guardato a lungo da ragazzo sulla costa algerina.

E ancora, con rapida approssimazione al «vicino» e altrettanto rapido riconoscimento della barriera, del muro che esclude:

Fra il mio paese e il mare si frappone una rupe, un agglomerato di ciottoli e conchiglie (o piuttosto di orme di conchiglie) dall'aspetto arcigno. La vegetazione di ginestre spinose, quelle che ha utilizzato Sutherland in "Capo di Spine» per dare un'idea della crudeltà del mondo, di cisti vellutati e fragili, di qualche ulivo superstite che vive a stento. Di lassù si gode, saltate le orrende costruzioni della nostra costa, di un vasto arco luminoso. La giornata era tersa, il mare mosso; l'acqua viaggiava e l'Esterel lontano prendeva il largo con le sue cime evanescenti; le due isole Sainte-Margherite e Saint-Honorat sembravano anch'esse velieri d'argento. Ma non riuscivo a trasognarmi, a comporre in pace quel paesaggio.

\section{Per terminare così:}

Ma, sogni a parte, non so veramente che dire, questo azzurro che scolpisce le cose che tocca e le corrode, che ha sovrastato un mondo di pastori, di pescatori, di ulivicoltori, è pieno di ombre segrete sempre più fonde per eccesso di storia e di luce.

A parte la solita "apertura a destra» (orografica), dal suo lembo di terra non verso la costa italiana, ma verso il ponente francese, subito il mare, le due isole di Cannes e i monti (l'Esterel), il Mediterraneo non costituisce una autentica realtà geografica che assuma poi la configurazione simbolica di uno spazio dotato di valori in sé, componga insomma la figura di un ideale, sia pure quello sotteso al "passaggio" (il rito di ogni frontiera versus l'avvertenza del confine come barriera, impedimento: «Fra il mio paese e il mare si frappone una rupe $[\ldots] »)$, non una realtà storica per eccesso di storia, non un partecipabile bacino sacrale - come potrebbe essere quello che contiene una frontiera di religioni affini e avverse intersecantesi — per deliberata esclusione degli déi 
greci e del dio cristiano, il Mediterraneo è per lui semplicemente ma coraggiosamente - il coraggio della via di togliere — l'abbacinante specchio d'acqua dirimpettaio, il contraltare laico della terra nascosta, dell'asylum contornato di ginestre spinose sutherlandiane e di ulivi stenti. ${ }^{26}$

Staticità assoluta? No, in apparenza. L' Hondurian star, la nave di Edoardo in Attesa, addirittura risale il maestrale, vento che travalica i confini, ne raggiunge il supposto, fantasticato nido a Saint-Malo e poi scende a Tolone, in Provenza, così come dalla sua terra Biamonti fruga con lo sguardo e ascolta le balze che scendono in Francia. Il punto di vista è quello di un confinato in un «porto» di altura montana, e il quadro paesistico pare rielaborato sull'onda della condizione «trasognata» in studio con la luce rivissuta come un soffitto schiacciante, come un'epidermide. Ė il paesaggio del prigioniero nel villaggio sulle alture, il porto con un unico, finalmente autentico marinaio tra gli ulivi.

7.

Chi ha parlato di «pathos della frontiera» è Italo Calvino nella quarta di copertina, battesimale e ormai famosa, del primo romanzo:

$\mathrm{Ci}$ sono romanzi-paesaggio così come ci sono romanzi-ritratto. Questo vive pagina per pagina, ora per ora, della luce del paesaggio aspro e scosceso dell'entroterra ligure, nell'estremo suo lembo di Ponente, al confine con la Francia. [...]

Tra i casolari di pietre e i villaggi di bungalow, i due aspetti della Riviera sono qui presenti insieme: un'agricoltura faticosa e solitaria e il mondo facile del turismo, a cui s'aggiunge la nuova dimensione del vagabondaggio giovanile che segue il miraggio della droga. E poi il pathos della frontiera, con la sua drammaticità depositata in tante storie di guerra, di contrabbando, d'espatrii clandestini.

Quando usa il termine «frontiera», Calvino, che vuole indicare? Un sinonimo di confine? Pare proprio di sì; visto che aggiunge le categorie di guerra,

26. Del resto le cognizioni e definizioni geografiche di Biamonti nelle grandi scale si deformano subito nel momento in cui vengono adibite a definire, simbolicamente, uno stato d'animo, dove il psicologico e il culturale si fondono: «Sia il golfo di Genova che il golfo di Marsiglia sono due lezioni di lucidità, di luminosità e di lucidità. Lo è anche il golfo di Orano, da dove è partito Camus: le prose nord-africane di Camus (Noces, L'été) sono bellissime. Questa lucidità mediterranea, di un pensiero che trova in sé la nascita e il compimento, che non rimanda a nessun aldilà, che fa meditare solo sull'aldiquà ("oh anima mia esaurisciti in un compito mortale!"), viene dalla Grecia. Mi è servito molto questo parametro per giudicare le cose e la vita.» («Intervista», in "Il paesaggio come compensazione» [...], cit., p. 52). Chissà cosa intende Biamonti per "Golfo di Genova", climaticamente, meteorologicamente, luministicamente, antropologicamente diversissimo da quello di Marsiglia tanto quanto è distantissimo dall'acqua salata che sta tra Ventimiglia e Mentone. È che la geografia biamontiana inchioda alla toponomastica delle mappe cartigli culturali che fanno riferimento a un universo intimo, nella stessa misura in cui al privilegiato descrittivismo minuto, topografico, s'aggancia la vibrazione dello status, psicologico e simbolico, di un personaggio. 
contrabbando ed espatrio clandestino. Comunque non si occupa, né preoccupa di ciò che irretisce noi, e non solo leggendo Biamonti: la divaricazione antropologica sottesa ai due termini quando non li si voglia assumere banalmente come equivalenti. Tra una linea stabile che divide in modo sempre uguale e che può costituire, certo, un ottimo punto di osservazione e riflessione, e una fascia mobile che si sposta, avanza e indietreggia, ingloba e abbandona. Il confine, invece, ha in sé una carica, una forza ad escludendum. S'intende che le due categorie vengono qui assolutizzate a bella posta e con una precisa intenzione euristica. E che il confine di Biamonti non coincide certo con il confine doganale.

Ne parleremo in seguito: ma è chiaro che il termine "frontiera» va misurato anche sugli episodi storici che lo inverano. Non si dirà di quello oltreatlantico, di qualità antonomasica che ha generato, appunto, il mito o la leggenda della Frontiera, assolutamente inesportabile; ma, nell'ambito mediterraneo e italiano, restando circoscritti alla cultura letteraria, dell'area del Nord-Est, di cui si potrà cogliere il carattere di «Marca di frontiera» (Magris), ovvero della Trieste novecentesca e della regione che la circonda dove gli appartenenti a una etnia e a una lingua si scambiano idealità nazionali e linguistiche e mirano sempre a un «altrove». ${ }^{27}$ Riprenderemo i raffronti tra non molto.

Non che alcuni di questi caratteri socioculturali siano assenti in Biamonti, ma in lui sembra che il fermento e il perenne status agonistico della frontiera abbiano trovato una definitiva composizione, per cui, infine, ogni elemento ritorna al suo preciso luogo deputato. Uomini e cose tornano al loro suolo e camposanto.

Non è escluso, sia chiaro, che ci possa essere una qualche nostalgia di un paese, di una grande Provenza o della civiltà francese (però, intanto, non c’è opzione precisa e decisiva tra Provenza e Francia), ma le eventuali aspirazioni non sono che un elemento stabilizzato, oramai traguardato nella specola di chi sta piantato nel suo orto, diviso da una roccia, quasi leopardiana siepe, per accennare così ad un altro autore a lui carissimo.

8.

Se, come abbiamo visto, il Mediterraneo non costituisce "frontiera», e offre invece le coordinate esterne per la disposizione di scelte letterarie e culturali (Montale, Camus) e religiose (il fare a meno degli déi), e anche il lembo di Provenza putativa vive e alimenta nello scrittore la serie delle contrapposizioni fondamentali, si dirà allora che la geografia e i suoi nomi sono principalmente in funzione delle figure dell'opposizione e dell'esclusione, della separazione degli elementi primigenii. Propriamente non c'è navigazione. Allora, in modo conseguente, gli effetti di paesaggio e di luce incarnano il principio di divisione. Basti qualche prelievo, a cominciare dal primo romanzo: 
Il crinale vibrò nel sole, come un maroso artigliato dal vento.

Si ricordò che sul mare talvolta gli era parso di vedere il crepaccio del mondo - malinconie, oh solo malinconie senzaltro - il crepaccio entro cui il mondo spariva. L'onda di roccia invece proteggeva: ferma lassù, piena di luce.

Mare e terra sono due mondi separati, l'uno è l'abisso, l'altra è la nicchia. Nella introiezione dei due mondi, la coscienza di Gregorio il contadino-marinaio (o finto marinaio: tutti i marinai di Biamonti, diciamolo, sono un po' improbabili) è dimidiata, patisce il «male del ferro» e lascia «il mare al suo abisso", il mare che ossessiona "proprio per il suo sciogliersi nell'eterno e nel nulla». Anche figurativamente, in quella sorta di ekfrasis di un quadro virtuale che dà l'impressione di essere, — ma non è precisamente — la pagina di Biamonti, ${ }^{28}$ si stagliano due regni opposti:

Una luce radente spianava il mare e lo sollevava nelle insenature; anche al largo esso si alzava sino a cozzare contro il cielo. Un altro mare, d'ombra, scendeva dalle catene rocciose.

Piuttosto che la spatola "costruttiva» (c'è chi lo ha definito un architetto del paesaggio) di Cézanne, Biamonti usa il bisturi della luce dimidiante, l'universo che lo circonda ne riemerge scisso, di netto. ${ }^{29} \mathrm{E}$ dessa, la luce, che costruisce il confine simbolico: tra luminosità della vita e non riscattabile opacità della morte.

Così come non c'è riscatto per i morti. Sogni pure la donna, Laurence in questo caso, un luogo di incontro:

La sera, il tramonto solenne, sulla «Baia degli angeli», le fece di nuovo sognare la terra di nessuno dove i morti e i vivi si potevano incontrare, dove Jean-Pierre poteva comparire. ${ }^{30}$

28. Dopotutto si tratta di scrittura, e qui lo stile surroga con un breve ma mirato arsenale di figure retoriche - regina la sinestesia - ciò che la pittura rende con i rapporti timbrici e con il "costruttivismo" generale del quadro.

29. L'influsso della pittura di paesaggio è indubbiamente fortissimo nei libri di Biamonti, forse non occorre esagerarne la portata diretta, ovvero pensare che lui, scrivendo, abbia avuto sempre o quasi, necessariamente, un preciso dato artistico-figurativo in mente. Anche la pittura fa parte di un bagaglio culturale, mentale, percettivo a lungo ruminato, che poi si scioglie in una prosa tutta sua vicina al lirismo e fitta di sofisticate figure retoriche. Ebbe tra l'altro a dichiarare lo stesso interessato: «Inoltre non si scriva che il mio incontro con Morlotti o con altri pittori è stato determinate per me, come ha proposto nel suo schema per una possibile biografia. Quando ho conosciuto Morlotti avevo già le mie idee sulla pittura. Anzi, siamo diventati amici perché le nostre idee sulla pittura erano collimanti. È dall'età di diciott'anni che ammiro Cézanne.» («Intervista», in "Il paesaggio è una compensazione» [...], cit. p. 50). Tuttavia è utilissima la ricognizione sul retroterra pittorico di Biamonti; non solo per ciò rinvio alla densa ricostruzione di Paolo ZUBLENA, «Lo sguardo malinconico sullo spazio-evento. Biamonti, Morlotti e il paesaggio dipinto", in Marcello CiCCUTO (a cura di), I segni Incrociati II. Letteratura Italiana del '900 e Arte Figurativa, Viareggio-Lucca: Mauro Baroni editore, 2002, p. 427-457.

30. Francesco Biamonti, L'angelo di Avrigue, cit., p. 87. 
Rimane intatta l'impossibilità di quello scambio escatologico che crea la frontiera tipicamente mediterranea della nekuia (da Omero a Virgilio a Pascoli a Montale: la zona sacra, invisibile e indeterminata del colloquio tra vivi e morti, e dello scambio delle parole, dei rimpianti, promesse, memorie, resipiscenze). I segni che testimoniano gli ultimi istanti della vita di Jean-Pierre stanno fuori del cerchio della morte: «No, non dentro l'alone della morte, ma completamente fuori». Se il luogo è marchiato dal confine, questo può divenire il crisma che gli impedisce di degradarsi a non-luogo, ciò che può restituirgli almeno la dignità della «soglia» degli antichi, proprio nel momento in cui il passo sta per travalicarlo:

Dal cielo laggiù si schiodava una luce severa. Cadeva sulle gobbe della rupe dove Jean-Pierre si era avvicinato al pauroso confine. $\mathrm{E}$ ancora una volta tentò di rivedere quel volto posato sul capezzale roccioso, che diffondeva sulla pietra funerea una gioia rude, come se avesse varcato le tristi soglie, il cuore liberato da un antico terrore. ${ }^{31}$

L'arte di Biamonti vive in fondo di questa qualità ossimorica, la sacralità del mondo, cui s'aggrappa con pervicacia, non perviene dall'oltre-confine, non riceve nessuna giustificazione o investitura dall'oltre, ma s'impone - quando riesca - proprio nel duro confronto con il crepaccio, l'abisso netto e vertiginoso che da ogni parte ci divide.

9.

A forza di marcare il pittoricismo di Biamonti insieme con i debiti, — da lui stesso con piacere confessati — , con Cézanne e i postimpressionisti, si è dimenticato che in letteratura tutto è "effetto di». Almeno in partenza, niente di più distante dalla pittura che la parola, ovvio. I prelievi dei maggiori passi «pittorici» forniscono dunque la verifica del processo di scarnificazione, disarticolazione del paesaggio, della sua frantumazione o decostruzione attraverso la luce. Quasi mai, infatti, siamo di fronte a un vero, compiuto paesaggio quale quello cui i pittori di riferimento ci hanno abituati. Anche questo sarà da meditare sulla via del pittorico e del simbolico: la luce che fu l'unità del paesaggio ora ne è il bisturi. Non c'è quasi mai il momento di ricostruzione materica, sulla pagina, di un analogo della visione armonica e complessiva di colui che raffigurò in mille toni la Montagne-Sainte-Victoire. La luce, in Biamonti, mentre lo accenna quasi lo distrugge, il paesaggio, e tende a surrogarlo. ${ }^{32}$

Non c'è luogo in cui essa si posi la che non venga segnato da un discrimine, da una linea affilata. La luce disegna i confini orografici («solo un luccicore listava il crinale»). ${ }^{33}$ E siamo nell'ultimo libro, Le parole la notte. Se è così 
radicato nella cultura estetica di chi scrive, forse il "confine» può aspirare a essere inserito dentro un paradigma più ampio, antropologico, naturalmente in seconda battuta e in quanto tradizione collettiva che sta alle spalle più che come scelta personale (Biamonti fu semmai un bibliotecario): è poi, infatti, anche il confine ligure del contadino, sorvegliato e conteso nelle "liti di confine» accennate da Biamonti, nei "diritti dei passaggi agresti», insomma la Liguria delle "pietre confinarie». Come ogni cosa localistica e vernacola assurge a una sua carica semantica rivelatrice, più generale, che infine abbandona ogni riferimento sociologico. Qui si offrono i connotati, appunto, di un uomo di confine che, come tutti noi, non ha più il senso della frontiera (a cui, solo eventualmente, accediamo per altra via, magari nel mondo cibernetico, virtuale). Lo hanno gli immigrati, loro sì. Dal loro punto di vista il Mediterraneo e l'Europa intera attraverso le numerose porte rischiose e nascoste (una è quella nei pressi della quale viveva e scriveva Biamonti e agisce il passeur di Vento largo) è un'immensa new frontier. Perciò l'uomo di confine e gli uomini della frontiera non si incontrano mai davvero, e sulla pagina solo di sfuggita.

Nel quadro di una situazione e condizione di confine e di confinato, la luce resta la vera protagonista dell'ultimo romanzo in rapporto con il tempo delle cose e degli uomini. Nel rapporto, essenziale, con la morte. Il mondo di Biamonti è un mondo in cui le nude e opache pietre (le rocce o i frammenti di insediamenti diroccati), vivono di luce ("pietra nuda con pilastri come colonne lucenti», «le pietre brillavano») ${ }^{34}$ e dove, viceversa, la vita e i corpi umani — in ispecie quelli femminili — si pietrificano e si mineralizzano.

10.

E' persino possibile che un tale paradigma si possa estendere ad alcuni aspetti della più alta fucina ligure delle due Riviere, non a caso quella poetica. Almeno due.

Non certo in termini storico-culturali, e neppure in termini puramente intimistico-esistenziali, ma in una riconfigurazione poetica affatto personale che tutti li riassume e li travalica, chi ha espresso nel modo più energico e definitivo l'esperienza di confine (decisamente non di frontiera), connotandola in senso riservatissimo, è Montale. Dentro la cifra del paesaggio degli Ossi, il movimento - tentativo augurio tensione - dalla terra verso il fuori, verso il mare, non è un'escursione orizzontale, come ci si attenderebbe, ma s'instaura nella dimensione dell'alto e del basso. Ciò che poi si para davanti, le onde, sono vortici, trombe, risucchi (e persino «scaglie» rilevate): non uno dei tratti realistici e metaforici della verticalità va perduto. E il mare, visto dall'alto, non può essere scorto che nel suo palpito o nel suo delirio, cioè nel suo lievitare e nel suo spasmodico e caotico sollevarsi. Anzi, l'intera avventura degli Ossi può condensarsi nella staticità di un soggetto che nel suo "estremo angolo d'orto" sente fortissimamente l'invalicabiltà del limite e si protende superiormente 0 
protende il tu con cui l'io-personaggio fonda il decisivo dialogo (proprio sul tema del moto dentro il paesaggio) verso l'alto: "Il canneto rispunta i suoi cimelli / nella serenità che non si ragna: / l'orto assetato sporge irti ramelli / oltre i chiusi ripari, all'afa stagna» (poi "un albero di nuvole sull'acqua / cresce», infine "tutto divaga / dal suo solco, dirupa, spare in bruma»: s'innalza verso l'alto, l'albero di nuvole, poi il paesaggio dirupa, crolla in basso). Così i "pochi stocchi d'erbaspada / penduli da un ciglione / sul delirio del mare». Così «ora son io / l'agave che s'abbarbica al crepaccio / dello scoglio / e sfugge al mare da le braccia d'alghe / che spalanca ampie gole [...] sento / la mia immobilità come un tormento.» 35

L'altro è, — s'è già indovinato — , Caproni.

Non controcantava in debutto: "Non nel mezzo, ma al limite / del cammino"? (corsivo mio). Non si ripete, la figura, in modo altrettanto esibito, altrove?

\section{Raggiungimento}
Andavo. Andavo
Cercavo dove poter sostare.
Ero ormai sul discrimine.
Dove finisce l'erba
e comincia il mare. ${ }^{36}$

Non si colloca sempre, colui che incede o guarda, «al limite», fin dall' Antefatto del Franco cacciatore: "Sedetti fuor dell'osteria, / al limite della foresta»?37

Non condividono, la «finestra» e la "porta», per certo verso la sinonimia col «confine»? E così «barriera», "dogana»?

Da questo punto di vista si potrebbe puntare a una definizione di Caproni come uomo e paesaggista di confine.

35. Alla fine degli Ossi l'incontro (solo il «cenno») con «una vita strozzata per lui sorta», cioè con una labile apparizione femminile, che si schiude da un paesaggio non più riconoscibile come l'originario e consueto (non più il quasi geometrico orto coi muri e la rete, ma un paesaggio di riviera molto più fermentante, inquieto e luministico, allucinato se non fosse puntualmente realistico, che di colpo s'allarga in un campo visivo cosmicamente espanso: «e il vento / la porta con la cenere degli astri») sottende l'antica originaria esperienza del prigioniero immobile, il quale persino rivendicava la qualità terricola di chi risiede e non può che rimanere abbarbicato al di qua di una linea netta e ben solidamente marcata: Esterina è «sommersa» dalle onde, poi "esce» dal fiotto, poi si «alza» e "s'avanza» sul trampolino e, alla fine del moto ascendente, «s'abbatte» giù «fra le braccia / del suo divino amico che l'afferra", il mare. "Ti guardiamo noi, della razza di chi rimane a terra».

36. Giorgio CAPRONI, Il Conte di Kevenhüller, in L'Opera in versi, ediz. critica a cura di Luca Zuliani, Milano: Mondadori, 1998, p. 649.

37. Ibid., p. 395. 


\section{1.}

Abbiamo fatto di tutto perché il lettore straniero - e non solo lui — abbia difficoltà, a questo punto, ad impiegare per la Liguria la categoria di frontiera. Non si dice quella d'invenzione americana, ma quella di origine europea, che proprio nel triestino ha una delle sue più alte incarnazioni. «Marca di frontiera» (Magris), Trieste e quella più vasta area battezzata dall'Ascoli Venezia Giulia è dall'origine realtà plurinazionale, zona relativamente franca dove ciascuna «etnia» guarda altrove: prima della Grande Guerra gli italiani guardano all'Italia, gli austrotedeschi all'Oltralpe, a Vienna, gli slavi al risveglio delle loro genti. La qualità elastica di una frontiera culturale ed etnica che s'espande e si contrae, che pulsa, è insita nelle opere che vi allignano e iscritta persino nei nomi degli autori. Si allude appena pro memoria, e tanto per essere chiari, al fatto che il commerciante-industriale Ettore Schmitz appartiene al mondo economico austriaco, mentre Italo Svevo si trasferisce nel mondo culturale italiano: tale conflitto fa aggio su quello città-campagna. Più tardi un simbolo della funzione mediatrice e degli scambi e smistamenti di culture (persino extraeuropee, orientali) sarà quello di un tipico «intellettuale di frontiera», il glossatore poliglotta, amico di Montale, destinatario di alcune sue lettere in versi e prosa, Bobi Bazlen. ${ }^{38} \mathrm{Ma}$ il discorso portato fino ai nostri giorni si farebbe troppo lungo per poter arrivare alla generazione del medesimo Claudio Magris.

Certo, la situazione storica e geopolitica del confine occidentale non è neppure paragonabile a quella d'oriente, alla Trieste del 1954, cioè a un territorio libero amministrato dagli americani e dagli inglesi, che faceva e non faceva parte dell'Italia. Simili paragoni hanno solo una funzione didascalica, ma servono pure per verificare le proporzioni. Per cui tanto vale ascoltare lo stesso Magris con un poco di agio. Rammentato il fondamentale imprinting degli intellettuali mitteleuropei indotto dall'Impero austro-ungarico, per cui scrittori polacchi giunti sulla riva sinistra del Danubio di fronte a Belgrado (l'antica frontiera della monarchia) si sentono ancora in patria su quella sponda avvertita come il proprio mondo e considerano la sponda opposta, Belgrado appunto, come l'inizio di un altro, Magris passa a ricordare l'immediato dopoguerra, quell'anno fatidico 1954 :

La frontiera triestina è e soprattutto era una frontiera con l'Est; quella che vedevo concretamente davanti a me quando andavo a giocare sul Carso con i miei amici, era la Cortina di ferro, la frontiera che tagliava in due, allora, il mondo intero e che correva a pochissimi chilometri da casa mia [...]. Ma, contemporaneamente, quelle terre al di là del confine, che appartenevano all'altra Europa, erano state italiane sino a pochi anni prima, sino alla fine della guerra, quando erano state occupate e annesse dalla Jugoslavia [...]. Oltre quel confine cominciava l'altra Europa - questo aggettivo «altra» derivava certo

38. Vedi A. Ara e C. Magris, «Trieste e la Venezia Giulia», in A. Asor Rosa (diretta da) Letteratura italiana. Storia e geografia, vol III; "L'età contemporanea», Einaudi: Torino, 1989, p. 797-839. 
in primo luogo dall'appartenenza all'universo staliniano, ma marcava pure un'ignoranza da parte occidentale. Anch'io, da ragazzo, credevo che Praga fosse a est di Vienna ed ero rimasto un po' stupito davanti alla smentita dell'atlante scolastico [...]. La frontiera è ponte o barriera; stimola il dialogo o lo soffoca. La mia educazione sentimentale è stata segnata dall'odissea dei confini, dalla loro arbitrarietà e inevitabilità. A ciò appartiene ad esempio la definizione, che in quegli anni accadeva di sentire spesso, di Trieste quale "piccola Berlino» [...]. Si aveva talora la sensazione non soltanto di vivere su una frontiera, ma di essere una frontiera [...]. A Trieste tutto ciò produceva spesso un sentimento di incertezza, di inappartenenza ed estraneità; un contraddittorio sentimento di vivere al centro e insieme alla periferia della vita. [...] La coscienza collettiva si sentiva soffocata da ogni parte da confini, ma si circondava a sua volta febbrilmente da nuove frontiere, per sfuggire a ogni precisa appartenenza e per costruirsi un'identità grazie a questa alterità esasperata. Una città italiana, che aveva vissuto intensamente la sua passione nazionale e i cui patrioti portavano spesso nomi d'origine tedesca o slava, come a Praga i nazionalisti tedeschi dai cognomi cechi e viceversa. Oppure come i capi dell'irredentismo croato in Dalmazia, che nel secolo scorso si riunivano al caffé Muljacic a Spalato e formulavano in italiano i programmi delle più accese rivendicazioni croate. Una città che si sentiva italiana in modo così particolare, da considerarsi spesso incompresa dal resto della nazione e da ritenersi dunque l'Italia più autentica - come se al di là dell'Isonzo, altro confine fondamentale sulla carta geopolitica e fantastica - , cominciasse l'Italia ufficiale e dunque meno vera. ${ }^{39}$

\section{2.}

Per finire proponiamo un altro esperimento, visto che abbiamo fatto ricorso a molte comparazioni e raffronti. Pochi concetti quanto quelli di «confine» e «frontiera» reclamano dal loro nucleo con altrettanta forza distinzioni e definizioni per antitesi.

Si possono fare altri confronti. Anche geograficamente estremi. Non solo per semplice curiosità si può partire dal seguente lacerto:

Nella neutra austerità di quel terreno, tutti i fenomeni erano affidati a una strana eguaglianza, e nessuna cosa, né un ragno, né una pietra, né un filo d'er$\mathrm{ba}$, poteva vantare diritto di precedenza. L'assoluta visibilità di questi oggetti snaturava la loro familiarità, poiché l'occhio identifica la totalità sulla base di qualche caratteristica o parte, mentre qui nulla era più luminoso di qualcos'altro e nulla era più adombrato, e nella democrazia ottica di paesaggi del genere qualsiasi predilezione è pura bizzarria, e fra un uomo e una roccia si creano parentele impreviste. ${ }^{40}$

39. Claudio MAGRIS, "Dall'altra parte. Considerazioni di frontiera», in ID. Utopia e disincanto. Saggi 1974-1998, Milano: Garzanti, 1999, p. 51-54.

40. Cormac MCCARThy, Meridiano di sangue o rosso di sera nel West, Torino: Einaudi, 1996, p. 254. [ed. orig. Blood Meridian Or the Evening Redness in the West, Alfred A. Knopf, 1985]. 
C'è un unico elemento che denuncia manifestamente l'appartenenza di questo brano giammai a un europeo, ma a un americano: il richiamo alla «democrazia ottica» con quel che ne consegue. Eppure questi elementi ricordano Biamonti, incredibilmente. Si tratta - i lettori affezionati l'avranno riconosciuto subito senza ricorrere alla nota - di Cormac McCarthy, Meridiano di sangue. Soprattutto con la "trilogia della frontiera" (Oltre il confine, Cavalli selvaggi, Città della pianura ${ }^{41}$ MacCarthy ha fatto rivivere letterariamente il mito americano della frontiera imprimendole, intanto, una rotazione geografica dal tradizionale Ovest al Sud-Ovest del border-line tra Stati Uniti e Messico e declinandolo nelle forme moderne di uno scontro violentissimo e cruento tra uomini e cose, in cui il paesaggio di frontiera non è semplice testimone muto ma personaggio interlocutore sempre teso e straziante, l'enigmatico totem su cui i protagonisti raffrontano le loro azioni e pensieri:

Scrutavano il paesaggio come se per loro rappresentasse un problema, qualcosa che non avevano ancora deciso. ${ }^{42}$

Benchè mutata, e quanto, dall'epoca aurea del western, nello scrittore di El Paso la frontiera è ancora conflitto, duello, selezione naturale e «formazione» del giovane picaro, cavaliere errante che lascia la terra per avviarsi alla scoperta di un'altra. Il viaggio non ha nulla di pretestuoso, nonostante le concessioni alla descrittività e alle riflessioni gnomiche che interrompono l'avventura, a volte si direbbero sul punto di paralizzarla; così come resiste l'antica fiducia nelle storie e nelle avventure che s'intrecciano attorno ai border-lines dense, vive e popolari; non smagrite e pallide, narratologicamente frammentate, dia-

41. Cormac MCCARTHY, Oltre il confine, Torino: Einaudi, 1995 [The Crossing, Alfred A. Knopf, 1994]; Cavalli selvaggi, Torino: Einaudi, 1996 [All the Pretty Horses, Alfred A. Knopf, 1992]; Città della pianura, Torino: Einaudi, 1999 [Cities of the Plain, Alfred A. Knopf, 1998]. Non solo il formidabile rilievo accordato al paesaggio, ai toni, colori della terra e del cielo, persino il pessimismo improntato a una laica teodicea senza speranza può essere accostato per un confronto. Biamonti: «- Il nostro [di marinai] è un lavoro di figli di nessuno. / — Un tempo era da figli di vedove, - Henri disse. - Adesso non si muore più, ma si va alla deriva - . E si mise a imprecare contro il viaggio che stavano facendo. / - Non imprechiamo, - Edoardo disse. - Cerchiamo di tornare in porto. / - Tutta la vita che non faccio altro $[. .$.$] con tutta la gente che se ne sta a terra. Il potere è strano, il mondo malvagio,$ concepito da un demiurgo inferiore sfuggito di mano al re della luce.» (Attesa sul mare, cit., p. 73). McCarthy: «Guardò le stelle compiere un semicerchio e morire nelle tenebre ai confini del mondo e sentì un'angoscia mortale trafiggergli il cuore. Immaginò la sofferenza del mondo come un parassita informe in incubazione che per svilupparsi cercava il calore delle anime umane. Credeva di sapere cosa rendeva gli uomini soggetti alle sue visite, ma quel che non sapeva ancora era che quella creatura era priva di mente e quindi incapace di conoscere i limiti dell'anima. Ed ebbe paura che l'anima potesse non avere alcun limite». (Cavalli selvaggi, cit., p. 256-257). Con le evidenti differenze, si capisce. In Cormac McCarthy c'è un paradossale senso trionfalistico dell'onnipotenza dell'animo umano che rende possibile un'epica del male puro, mentre in Biamonti tutto è trattenuto nel cerchio dell'elegia rassegnata della condizione statica dell'assediato dall'onnipervasività del male.

42. Cormac MCCarThy, Cavalli selvaggi, cit., p. 61. 
logicamente reticenti, offuscate dai richiami continui ai timbri e toni dell'ambiente e dalle mezze parole che aspirano al silenzio, come in Biamonti. ${ }^{43}$ Se nelle vicende dei giovani vagabondi di quello scrittore sta la forma di un apprendistato, nei vecchi del secondo è già tutto scritto nel libro: «Lo sa che il mondo è dominato dalla distruzione e dall'omicidio. Io non parlo. Se apro il libro non posso più fermarmi»; e pure in francese: "- Non mi faccia dire cose banali. Se apro il cahier de doléance non riesco più a chiuderlo.». ${ }^{44}$ Restano, formidabili affinità, imprevedibili, dentro una declinazione evidentemente diversissima dei temi del paesaggio e della frontiera.

\section{3.}

A ognuno il suo, sicuro. E a ogni generazione anche. Tra le tante divaricazioni dei meridiani geografici e culturali che la letteratura ha operato, Biamonti si staglia per la sua scelta di darsi nella sua schietta fisionomia particolare che declina una sottile dialettica interna, come abbiamo visto, tra frontiera e confine, ma sa con coraggio e onestà rinunciare a ogni mito (di religione del sacro, di religione del lavoro, alla Boine, del «tu» femminile, come in Montale, di patria putativa, e infine quello di una terra promessa culturale o politica, sia pur sognata e sofferta nei limiti imposti dalla condizione moderna); finalmente - e conseguentemente a una linea etica definita una volta per tutte — , si tiene stretto alla cocciuta fedeltà alla rupe, alla «fascia». Una fedeltà quasi drammaticamente verghiana se non passasse per un trattamento stilistico e poetico quasi opposto e anche per una struggente ricerca sottesa, una richiesta di «felicità» che percorre come un brivido tutta l'opera:

Chi nel passato aveva creduto in una qualsiasi forma di felicità terrena, al di fuori del possedere una casa in paese e una campagna rocciosa, si era perduto.

(Ed è il primo capitolo, seconda pagina, dell'Angelo di Avrigue).

43. I silenzi che contraddistinguono i dialoghi di Biamonti rappresentano il versante linguistico della figura antropologica dell'abitante del confine. Confine tra silenzio e linguaggio, tra comunicazione e chiusura, interruzione dei messaggi, soprattutto: «Nei recenti romanzi di uno degli scrittori più sensibili a questo tema, Francesco Biamonti, le virgolette, da sempre segno della parola diretta e viva che gli uomini si scambiano sulla pagina, racchiudono, invece, pensieri, parole dei protagonisti rivolte esclusivamente a se stessi, linguaggio mentale, intime considerazioni. Non sono neppure segni di un monologo ad alta voce, ma dialoghi muti, colloqui senza parole con se stessi, lingua anche visivamente senza interlocutori, afona. I personaggi di Biamonti si dicono non meno (e forse più) di quanto dicono, si chiedono non meno (e forse più) di quanto chiedono. Dialogano solo o soprattutto con se stessi.» (Vittorio COleTtI, Dietro la parola. Miti e ossessioni del Novecento, Alessandria: Edizioni dell'Orso, 2000, p. 59.).

44. Francesco BIAMONTI, Le parole la notte, cit., p. 75, 159. 